\title{
Evaluation of a New Integrated Fog Removal Algorithm IDCP with Airlight
}

\author{
Tarun A. Arora \\ Beant College of Engineering \& Technology (E.C.E), Gurdaspur, 143521, India \\ tarunbcet7869@gmail.com \\ Gurpadam B. Singh, Mandeep C. Kaur \\ Beant College of Engineering \& Technology, (E.C.E), Gurdaspur, 143521, India \\ gurpadam@yahoo.com
}

\begin{abstract}
This paper has proposed a new fog removal technique IDCP which will integrate dark channel prior with CLAHE and adaptive gamma correction to remove the fog from digital images. Fog in image reduces the visibility of the digital images. Poor visibility not only degrades the perceptual image quality but it also affects the performance of computer vision algorithms such as object detection, tracking, surveillance and segmentation.Various factors such as fog, mist and haze caused by the water droplets present in the air during bad weather leads to poor visibility. The proposed algorithm is designed and implemented in MATLAB using image processing toolbox. The comparison among Air-light and the proposed algorithm is also drawn based upon certain performance parameters. The comparison analysis has shown that the proposed algorithm has shown quite effective results.
\end{abstract}

Index terms - Visibility restoration, Air-light map, Fog removal and Haze.

\section{INTRODUCTION}

Visibility restoration refers to different methods that aim to reduce or remove the degradation that have occurred while the digital image was being obtained. The degradation may be due to various factors like relative object-camera motion, blur due to camera mis-focus, relative atmospheric turbulence and others. In this we will be discussing about the degradations due to bad weather such as fog, haze, rain and snow in an image. The image quality of outdoor screen in the fog and haze weather condition is usually degraded by the scattering of a light before reaching the camera due to these large quantities of suspended particles (e.g. fog, haze, smoke, impurities) in the atmosphere. This phenomenon affects the normal work of automatic monitoring system, outdoor recognition system and intelligent transportation system. Scattering is caused by two fundamental phenomena such as attenuation and air-light. By the usage of effective haze removal of image we can improve the stability and robustness of the visual system.

Haze removal is a tough task because fog depends on the unknown scene depth information. Fog effect is the function of distance between camera and object. Hence removal of fog requires the estimation of air-light map or depth map. The current haze removal method can be divided into two categories: image enhancement and image restoration. Image enhancement does not include the reasons of fog degrading image quality.

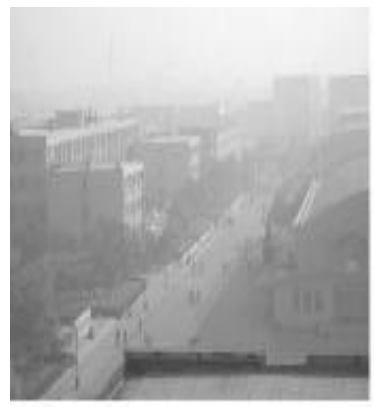

(a)

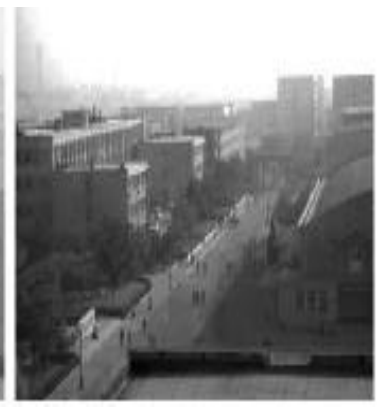

(b)
Fig 1: (a) Original image (b) Processed image

This method can improve the contrast of haze image but loses some of the information regarding image. Image restoration firstly studies the physical process of image imaging in foggy weather. After observing that degradation model of fog image will be established. At last, the degradation process is inverted to generate the fog free image without the degradation. So, the quality of degraded image could be improved.

\section{LITERATURE SURVEY}

Chen, et al. (2009) [1] has studied that in bad weather such as fog and haze can significantly degrade the visibility of scene. An iterative algorithm to adjust the color distortion affected by higher saturation using global and local rectifications was proposed. The working of technique like image matting and iterative matte optimization to treat the inherently similar pixels of some object and atmospheric light differently was explained. In this image is modelled as a linear combination of foreground image and background image by a map.

$\mathrm{Xu}$, et al. (2009) [2] has examined that images degraded by fog suffer from poor contrast. In order to remove this effect a contrast limited adaptive histogram 
equalization (CLAHE)-based method was proposed. To clip the histogram this method establishes a maximum value and redistributes the clipped pixels equally to each gray level. This algorithm is performed in three steps; firstly the color images captured by camera in foggy is converted from RGB (Red, Green and blue) color space to HSI space. The reason behind the conversion is the HSI (Hue, Saturation and Intensity) represents colors similarly how the human eye sense color. The HSI color represent attractive color model for image processing applications. Second, the intensity component of the image is processed by CLAHE. The hue and intensity remain unchanged. A new HSI image is obtained. Finally the new image in HSI color space is converted back to RGB color space to get the original image without degradations.

Wang, et al. (2010) [3] has explored that haze removal from the image depend upon the unknown depth information. This algorithm is based on the atmospheric scattering physics-based model. In this on selected region a dark channel prior is applied to obtain a novel estimation of atmospheric light. This model is based upon some observation on haze free outdoor image. The intensity of dark channel calculated gives rough approximation of the thickness of haze.

$\mathrm{Yu}$, et al. (2011) [4] has proposed a novel fast defogging method from a single image based on the scattering model. A white balancing is used prior to the scattering model applied for visibility restoration. Then an edge-preserving smoothing approach based on weighted least squares (WLS) optimization framework to smooth the edges of image. At last inverse scene albedo is applied for recovery process.

Shuai, et al. (2012) [5] discussed problems regarding the dark channel prior of color distortion problem for some light white bright area in image. An algorithm to estimate the media function in the use of median filtering based on the dark channel was proposed. After making media function more accurate a wiener filtering is applied. By this fog restoration problem is converted into an optimization problem and by minimizing mean square error a clearer, finally fog free image is obtained.

Cheng, et al. (2012) [6] has proposed a lowest channel prior for image fog removal. This algorithm is simplified from of dark channel prior. It is based on a key fact that fog-free intensity in a color image is usually a least value of tri-chromatic channels. In dark channel prior to estimate the transmission model it performs as a minimum filter for lowest intensity. This filter leads to halo artefacts, specifically in the neighbourhood of edge pixels. In this algorithm instead of minimum filter they utilises exact $\mathrm{O}(1)$ bilateral filter based on the raised cosines function to the weight value of neighbour to get fog-free image.

$\mathrm{Xu}$, et al. (2012) [7] has recommended a model based on the physical process of imaging in foggy weather. In this model a fast haze removal algorithm which is based on a fast bilateral filtering with dark channel prior is explained. Firstly, the atmospheric scattering model is used to describe the formation of haze image. Then an estimated transmission map is formed using dark channel prior. Then it is combined with gray scale to extract the refined transmission map by using fast bilateral filter instead of soft matting. The reason why the image is dim after the use of dark channel prior is observed and a transmission map formula is proposed to restore the color and contrast of the image.

Kang, et al. (2012) [8] has proposed a single image based rain removal frame work by properly formulating rain removal as an image decomposition problem based on MCA (morphological component analysis). Before applying a proposed method image is decomposed into low and high-frequency parts using a bilateral filter. By using sparse coding and dictionary learning algorithms the high frequency part is decomposed into rain component and non-rain component. Sparse coding is a technique of finding a sparse representation for a signal with a small number of nonzero or significant coefficients corresponding to the atoms in a dictionary. After this pre-processing step the proposed MCA (morphological component analysis)-based image decomposition to the $\mathrm{HF}$ part that can be further decomposed into the rain component.

Tripathi, et al. (2012) [9] has studied that fog formation is due to air-light and attenuation. Air-light increases the whiteness and attenuation increases the contrast in the scene. So a method is proposed which use bilateral filter to recover scene contrast and for the estimation of light. In this algorithm both pre and post processing steps are performed. Histogram equalization is used as pre processing to increase the contrast of the image prior to fog removal and also help to get better estimation of air-light map. Histogram stretching is used as post processing for increasing the contrast of fog removed image.

Tripathi, et al. (2012) [10] has proposed an algorithm which use anisotropic diffusion for estimation of air-light For color image RGB (red, green and blue) and HSI (Hue saturation and intensity) models are used.

Wei, et al. (2013) [11] has proposed a fast single image de-hazing algorithm based on the atmospheric scattering model. The proposed algorithm use numerical correction for the phenomenon. Firstly a dark channel phenomenon is applied via optical method, and approach for solving the parameter in atmospheric scattering is derived. Secondly a gray-scale opening operation and fast joint bilateral techniques, to calculate the global atmospheric light is used. Finally, to calculate the scene albedo (reflection coefficient) the model is inverted.

Im, et al. (2013) [12] has proposed a novel contrast enhancement method for backlit images that consists of three steps: i)computation of the transmission coefficients using the dark channel prior ii) generation of multiple images having different exposures based on the transmission coefficients and iii) image fusion. This approach first extracts under-exposed regions using the dark channel prior map, and then performs spatially adaptive contrast enhancement.

Kil, et al. (2013) [13] has recommended a de-hazing algorithm based on dark channel prior and contrast 
enhancement methods. They combined the advantages of these two conventional approaches for keeping the color while de-hazing process. An optimization function is proposed to balance between the contrast and colors distortion, where the contrast measure follows the conventional image statistics and the hue component is used to constrain the color changes.

Noh, et al. (2013) [14] has proposed a de-hazing method for single image on super-pixel domain. They applied Dark Channel Prior to the super pixel, instead on the conventional fixed size patch, to estimate the transmission map. They exploited quick shift algorithm to estimate the transmission map.

Long, et al. (2013) [15] has presented a simple, but effective, method for single remote sensing image haze removal. They used Dark Channel Prior to remove the effect of atmospheric light and roughly estimate the atmospheric veil. A low pass Gaussian filter was used to refine the atmospheric veil. To eliminate the color distortion image was recomputed.

Ullah, et al. (2013) [16] has observed that chromatic effects of image scattering can be reversed for retrieval of image information. The proposed model considers chromatic as well achromatic aspects of the image to define the Dark Channel prior. Contrast of the restored images is refined by further color fidelity. Dark channel is applied such that result can be obtained for both high saturated pixels and moderately saturated pixels. This model use the HIS color model which is intuitive based on the Intensity (I) and the Saturation (S) components of the foggy pixels.

\section{PROBLEM DEFINITION}

Main foundation of complications when processing outdoor images is the existence of the noise, haze, fog or smoke which reduces the quality of image by decreasing the contrast of the captured objects. This dissertation proposes a new improved algorithm and alternatives for visibility restoration from a foggy or low density images. The proposed algorithm will integrate dark channel prior, CLAHE and adaptive gamma correction to achieve the objective of this research work.

The main advantage is the probability to handle both color images and gray level images since the ambiguity between the presence of fog and the objects with low color saturation is resolved by presumptuous only small objects can have colors with low intensity. The algorithm is controlled only by a few parameters and consists in: atmospheric veil inference, image restoration and smoothing, tone mapping.

In order to performance comparison, different metrics of images and complexity theory will be considered. An appropriate comparison will be drawn among proposed technique and previous well known techniques.

\section{PROPOSED ALGORITHM (IDCP)}

Step I. Read the Input image

Step II. Now CLAHE on L*a*b color space operation will be applied to balance the effect of the light and colors.

Step III.Now Dark channel prior will come in action to reduce the effect of fog from digital image.

Step IV.Now adaptive gamma correction will be applied as a post processing operation to enhance the brightness of the system.

Step V. Now we will get the final image which has been visibly restored.

A. CLAHE on $\mathbf{L} * \mathbf{a} * \mathbf{b}$ color space:-Contrast limited adaptive histogram equalization short form is CLAHE. This method does not need any predicted weather information for the processing of hazed image. Firstly, the image captured by the camera in foggy condition is converted from RGB (red, green and blue) color space is converted to LAB color space. A Lab color space is a color-opponent space with dimension $\mathrm{L}$ for lightness and (a,b) for the color-opponent dimensions, based on nonlinearly compressed CIE XYZ color space coordinates.

B. Dark channel prior: - Dark channel prior is used for the estimation of atmospheric light in the de-hazed image to get the more proper result. This technique is mostly used for non-sky patches, as at least one color channel has very low intensity at some pixels. The low intensity in the dark channel is mainly due to three factors:-

i. Shadows(shadows of car, buildings etc)

ii. Colourful objects or surfaces(green grass, tree, flowers etc)

iii. Dark objects or surfaces(dark tree trunk, stone etc)

As the outdoor images are usually full of shadows and color-ful, the dark channels of these images will be really dark. Due to fog (air-light), a haze image is brighter than its image without haze. So we can say dark channel of haze image will have higher intensity in region with higher haze. So, visually the intensity of dark channel is a rough approximation of the thickness of haze.

C. Adaptive gamma correction:-A nonlinear operation used to code and decode luminance or tristimulus values in video or still image systems. Gamma correction defined by the following power-law expression:

$$
V_{\text {out }}=A V_{\text {in }}^{\gamma}
$$

Where $\mathrm{A}$ is a constant and the input and output values are non-negative real values; in the common case of $\mathrm{A}=1$, inputs and outputs are typically in the range $0-1$. A gamma value $\gamma<1$ is sometimes called an encoding gamma, and the process of encoding with this 
compressive power-law nonlinearity is called gamma compression; conversely a gamma value $\gamma>1$ is called a decoding gamma and the application of the expansive power-law nonlinearity is called gamma expansion.

\section{EXPERIMENTAL SET-UP}

In order to implement the proposed algorithm; design and implementation has been done in MATLAB using image processing toolbox. In order to do cross validation we have also implement the histogram equalization and non linear enhancement technique. Table 1 is showing the various images which are used in this research work. Images are given along with their formats. All the images are of different kind and each image has different kind of the light i.e. more or less in some images.

Table 1: Experimental images

\begin{tabular}{|c|c|c|}
\hline Name & Format & Size(KB) \\
\hline 1 & .jpg & 44.1 \\
\hline 2 & .jpg & 78.0 \\
\hline 3 & .jpg & 55.8 \\
\hline 4 & .jpg & 73.0 \\
\hline 5 &.$j p g$ & 23.2 \\
\hline 6 & .png & 919 \\
\hline 7 & .png & 331 \\
\hline 8 & .png & 349 \\
\hline 9 &.$p n g$ & 650 \\
\hline 10 & .png & 612 \\
\hline 11 & .png & 276 \\
\hline 12 & .png & 451 \\
\hline 13 & .png & 173 \\
\hline 14 & .png & 903 \\
\hline 15 & .jpg & 51.1 \\
\hline
\end{tabular}

\section{EXPERIMENTAL RESULTS}

For the purpose of cross validation we have taken 15 different images and passed to the CLAHE and proposed algorithm. Subsequent section contains a result of one of the 15 selected images to show the improvisation of the proposed algorithm over the CLAHE. For rest of the figures we had given the result in terms of parameter and they are compared to show the comparison between the IDCP and CLAHE.

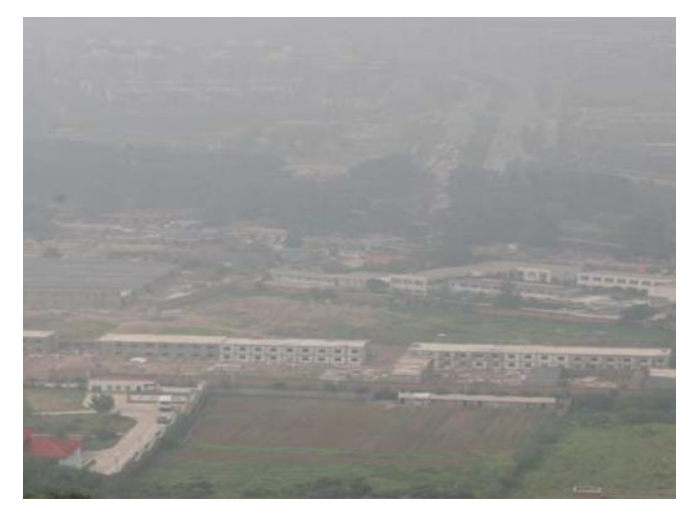

Fig 2 Foggy image
Fig 2 has shown the input image for experimental purpose. It has been clearly shown that the image contains a foggy image so the image is a foggy image, so the main objective is to remove it.

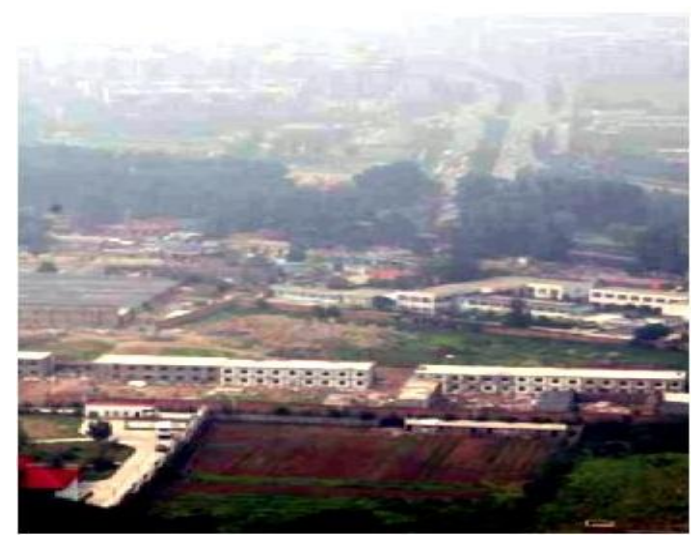

Fig 3: Air-light fog removed image

Fig 3 has shown the result of the existing Air-light algorithm. It has been clearly shown that the fog has been removed efficiently. But still some improvement is required to enhance the result further.

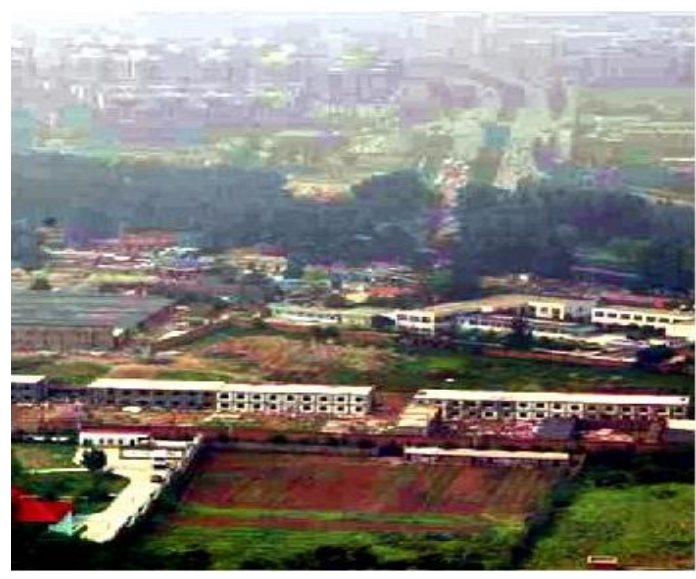

Fig 4: Proposed IDCP fog removed image

Fig 4 has shown the result of the proposed IDCP algorithm. It has been clearly shown that the fog has been removed more efficiently than image shown in figure 4.

\section{PERFORMANCE ANALYSIS}

This section contains the cross validation between existing and proposed techniques. Some well-known image performance parameters for digital images have been selected to prove that the performance of the proposed algorithm is quite better than the available methods.

Table 2 has shown the quantized analysis of the mean square error. As mean square error need to be reduced therefore the proposed algorithm is showing the better results than the available methods as mean square error is less in every case. 
Table 2: Mean Square Error comparison

\begin{tabular}{|c|c|c|}
\hline Image & Proposed & Air-light \\
\hline 1 & 2200 & 31700 \\
\hline 2 & 3620 & 20480 \\
\hline 3 & 3520 & 25850 \\
\hline 4 & 190 & 13240 \\
\hline 5 & 210 & 4150 \\
\hline 6 & 200 & 9330 \\
\hline 7 & 160 & 12620 \\
\hline 8 & 110 & 17590 \\
\hline 9 & 150 & 22500 \\
\hline 10 & 50 & 8800 \\
\hline 11 & 60 & 29530 \\
\hline 12 & 3020 & 19700 \\
\hline 13 & 250 & 22120 \\
\hline 14 & 240 & 10790 \\
\hline 15 & 9370 & 50330 \\
\hline
\end{tabular}

Table 3 has shown the quantized analysis of the Peak signal to noise ratio (PSNR). As PSNR need to be maximized therefore the proposed algorithm is showing better results than the available methods as PSNR is more in the case of IDCP.

Table 3: Peak Signal to Noise Ratio comparison

\begin{tabular}{|c|c|c|}
\hline Image & Proposed & Air-light \\
\hline 1 & 29.6295 & 15.9868 \\
\hline 2 & 15.6306 & 12.0795 \\
\hline 3 & 18.7055 & 10.4750 \\
\hline 4 & 30.9249 & 12.8124 \\
\hline 5 & 30.1627 & 15.2777 \\
\hline 6 & 30.4773 & 13.4669 \\
\hline 7 & 32.8009 & 9.7530 \\
\hline 8 & 39.2061 & 12.3253 \\
\hline 9 & 33.3717 & 11.3896 \\
\hline 10 & 54.2921 & 13.5830 \\
\hline 11 & 48.6955 & 8.5199 \\
\hline 12 & 16.1642 & 15.4564 \\
\hline 13 & 28.3293 & 11.9585 \\
\hline 14 & 28.7890 & 13.1875 \\
\hline 15 & 13.4581 & 7.4816 \\
\hline
\end{tabular}

Table 4 has shown the quantized analysis of the Geometric evaluation. As it need to be maximized therefore the proposed algorithm has shown the improved results than the available methods as geometric accuracy is more in the case of IDCP.

Table 4: Geometric Accuracy evaluation

\begin{tabular}{|c|c|c|}
\hline Image & Proposed & Air-light \\
\hline 1 & 99 & 84.7048 \\
\hline 2 & 84.1156 & 82.0321 \\
\hline 3 & 85.3502 & 81.0029 \\
\hline 4 & 99 & 82.8136 \\
\hline 5 & 99 & 86.2392 \\
\hline 6 & 99 & 82.9434 \\
\hline 7 & 99 & 80.8188 \\
\hline 8 & 99 & 82.7294 \\
\hline 9 & 99 & 81.4923 \\
\hline 10 & 99 & 82.4344 \\
\hline 11 & 99 & 80.5199 \\
\hline 12 & 92.1251 & 81.2548 \\
\hline 13 & 98.8998 & 82.0678 \\
\hline 14 & 99 & 82.6765 \\
\hline 15 & 82.6182 & 80.1691 \\
\hline
\end{tabular}

Table 5 has shown the analysis of the standard deviation. It is required to be minimized. It has improved results than the available methods as Standard deviation is either equal or less in the case of IDCP.

Table 5: Standard Deviation

\begin{tabular}{|c|c|c|}
\hline Image & Proposed & Air-light \\
\hline 1 & 0.0079 & 0.0094 \\
\hline 2 & 0.0094 & 0.0098 \\
\hline 3 & 0.0099 & 0.0099 \\
\hline 4 & 0.0062 & 0.0094 \\
\hline 5 & 0.0057 & 0.0087 \\
\hline 6 & 0.0082 & 0.0098 \\
\hline 7 & 0.0062 & 0.0098 \\
\hline 8 & 0.0054 & 0.0094 \\
\hline 9 & 0.0054 & 0.0098 \\
\hline 10 & 0.0051 & 0.0100 \\
\hline 11 & 0.0019 & 0.0097 \\
\hline 12 & 0.0099 & 0.0088 \\
\hline 13 & 0.0066 & 0.0096 \\
\hline 14 & 0.0064 & 0.0096 \\
\hline 15 & 0.0094 & 0.0100 \\
\hline
\end{tabular}

Table 6: Contrast Gain

\begin{tabular}{|c|c|c|}
\hline Image & Proposed & Airlight \\
\hline 1 & 21.0695 & 10.7637 \\
\hline 2 & 4.8883 & 3.9749 \\
\hline 3 & 7.0673 & 4.8276 \\
\hline 4 & 19.5040 & 9.8415 \\
\hline 5 & 21.3685 & 2.8275 \\
\hline 6 & 22.6652 & 12.5764 \\
\hline 7 & 19.1447 & 15.2516 \\
\hline 8 & 24.7825 & 11.3823 \\
\hline 9 & 24.9468 & 11.1800 \\
\hline 10 & 26.2248 & 8.2217 \\
\hline 11 & 20.6690 & 11.7348 \\
\hline 12 & 8.5805 & 3.2643 \\
\hline 13 & 17.3058 & 9.7510 \\
\hline 14 & 18.4754 & 6.0982 \\
\hline 15 & 3.1283 & 1.1611 \\
\hline
\end{tabular}

Table 6 has shown the comparative analysis of the contrast gain between the Air-light and the IDCP. The table has shown that the proposed algorithm has better contrast gain in every case. Therefore the proposed algorithm has shown significant improvement in result except one case.

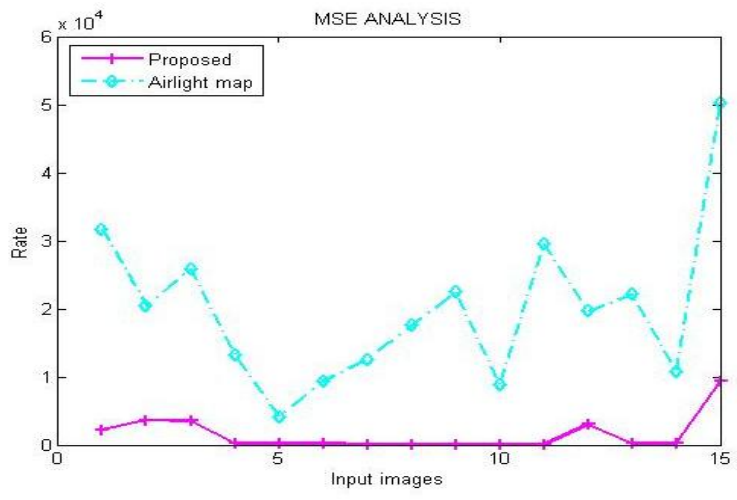

Fig 5: Mean Square Error comparison 
Fig 5 has shown the quantized analysis of the mean square error. As mean square error need to be reduced therefore the proposed algorithm is showing the better results than the available methods as mean square error is less in every case.

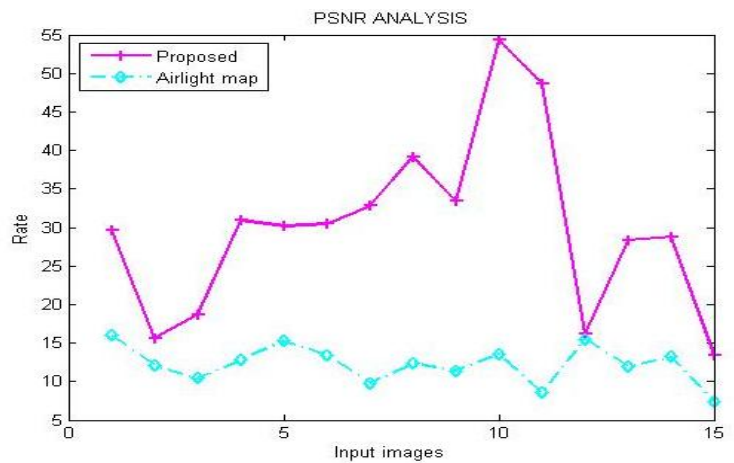

Fig 6: Peak Signal to Noise Ratio comparison

Fig 6 has shown the quantized analysis of the Peak signal to noise ratio (PSNR). As PSNR need to be maximized therefore the proposed algorithm is showing the better results than the available methods as PSNR is more in the case of IDCP.

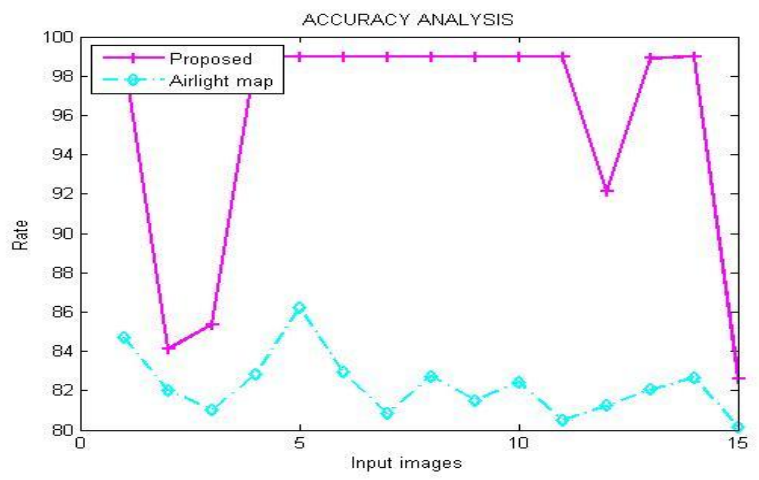

Fig 7: Geometric Accuracy evaluation

Fig 7 has shown the quantized analysis of the Geometric accuracy evaluation. As it need to be maximized therefore the proposed algorithm has shown the improved results than the available methods as geometric accuracy is more in the case of IDCP.

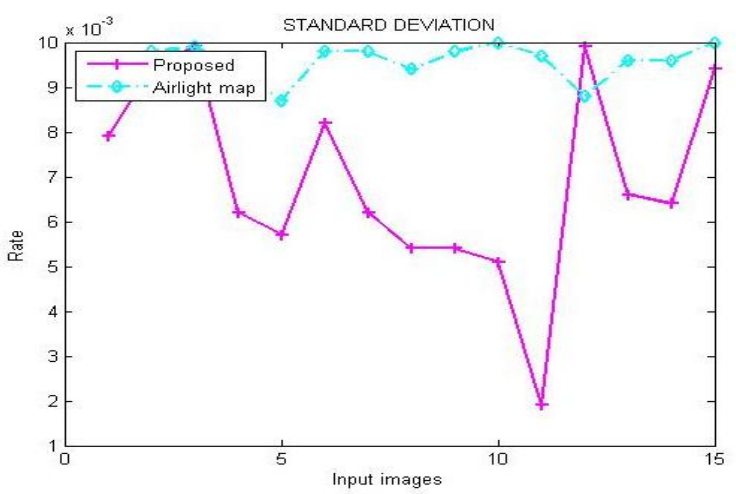

Fig 8: Standard Deviation
Fig 8 has shown the analysis of the standard deviation. It is required to be minimized. It has also shown significant improvement because in the most of the cases the proposed algorithm has minimized standard deviation. But in certain cases it is more but not much so it is also acceptable.

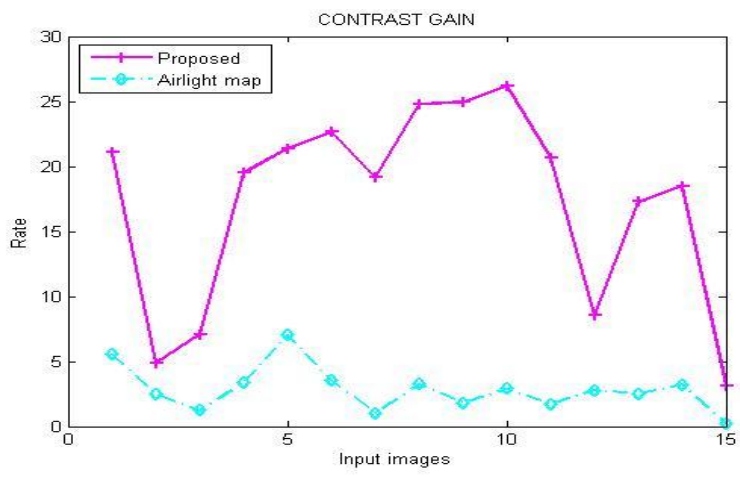

Fig 9: Contrast Gain

Fig 9 has shown the comparative analysis of the contrast gain between Air-light and the IDCP. The figure has shown that the proposed algorithm has better contrast gain in every case. Therefore the proposed algorithm has shown significant improvement in all case.

\section{CONCLUSION}

Fog removal algorithms become more useful for many vision applications. It is found that most of the existing researchers have neglected many issues; i.e. no technique is better for different kind of circumstances. The existing methods have neglected the use of gamma correction and histogram stretching to reduce the noise problem which will be presented in the output image of the existing fog removal algorithms. To reduce the problems of existing literature a new integrated algorithm has been proposed that has integrated the dark channel prior with CLAHE to enhance the results further.The proposed algorithm is designed and implemented in MATLAB using image processing toolbox. The comparison among Air-light and the proposed algorithm is also drawn based upon certain performance parameters. The comparison analysis has shown that the proposed algorithm has shown quite effective results. The main scope of the proposed algorithm is to improve the accuracy of the Intelligent Transportation System (ITS) especially when lane detection kinds of application come in action in VANETs. Therefore the proposed algorithm will become more useful in to preventing the road accidents as accidents rate is growing day by day due to poor driving and more traffic.

\section{REFERENCES}

[1] Chen, Mengyang, et al. "Single image defogging." Network Infrastructure and Digital Content, 2009. ICNIDC 2009. IEEE International Conference on. IEEE, 2009. 
[2] Xu, Zhiyuan, Xiaoming Liu, and Na Ji. "Fog removal from color images using contrast limited adaptive histogram equalization." Image and Signal Processing, 2009. CISP'09. 2nd International Congress on. IEEE, 2009.

[3] Wang, Yan, and Bo Wu. "Improved single image dehazing using dark channel prior." Intelligent Computing and Intelligent Systems (ICIS), 2010 IEEE International Conference on. Vol. 2. IEEE, 2010.

[4] Yu, Jing, and Qingmin Liao. "Fast single image fog removal using edge-preserving smoothing." Acoustics, Speech and Signal Processing (ICASSP), 2011 IEEE International Conference on. IEEE, 2011.

[5] Shuai, Yanjuan, Rui Liu, and Wenzhang He. "Image Haze Removal of Wiener Filtering Based on Dark Channel Prior." Computational Intelligence and Security (CIS), 2012 Eighth International Conference on. IEEE, 2012.

[6] Cheng, F-C., C-H. Lin, and J-L. Lin. "Constant time O (1) image fog removal using lowest level channel." Electronics Letters 48.22 (2012): 1404-1406.

[7] $\mathrm{Xu}$, Haoran, et al. "Fast image dehazing using improved dark channel prior." Information Science and Technology (ICIST), 2012 International Conference on. IEEE, 2012.

[8] Kang, Li-Wei, Chia-Wen Lin, and Yu-Hsiang Fu. "Automatic single-image-based rain streaks removal via image decomposition." Image Processing, IEEE Transactions on 21.4 (2012): 1742-1755.

[9] Tripathi, A. K., and S. Mukhopadhyay. "Single image fog removal using bilateral filter." Signal Processing, Computing and Control (ISPCC), 2012 IEEE International Conference on. IEEE, 2012.

[10] Tripathi, A. K., and S. Mukhopadhyay. "Single image fog removal using anisotropic diffusion." Image Processing, IET 6.7 (2012): 966-975.

[11] Wei, Sun, and Han Long. "A New Fast Single-Image Defog Algorithm." Intelligent System Design and Engineering Applications (ISDEA), 2013 Third International Conference on. IEEE, 2013.

[12] Im, Jaehyun, et al. "Dark channel prior-based spatially adaptive contrast enhancement for back lighting compensation." Acoustics, Speech and Signal Processing (ICASSP), 2013 IEEE International Conference on. IEEE, 2013.

[13] Kil, Tae Ho, Sang Hwa Lee, and Nam Ik Cho. "A dehazing algorithm using dark channel prior and contrast enhancement." Acoustics, Speech and Signal Processing (ICASSP), 2013 IEEE International Conference on. IEEE, 2013.

[14] Noh, Sang-Woo, Byungtae Ahn, and In So Kweon. "Haze removal on superpixel domain." Ubiquitous Robots and Ambient Intelligence (URAI), 2013 10th International Conference on. IEEE, 2013.

[15] Long, Jiao, et al. "Single Remote Sensing Image Dehazing." 1-1.

[16] Ullah, E., R. Nawaz, and J. Iqbal. "Single image haze removal using improved dark channel prior." Modelling, Identification \& Control (ICMIC), 2013 Proceedings of International Conference on. IEEE, 2013.

\section{Authors' Profiles}

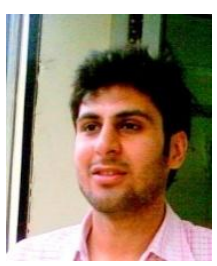

Tarun A. Arora was born on $2^{\text {nd }}$ may, 1988 at Gurdaspur, Punjab. I had done my B.Tech in Electronics and Communication of Engineering from Beant College of Engineering \& Technology, Gurdaspur, Punjab. I am pursuing my M.Tech in Electronics and Communication of Engineering from Beant College of

Engineering \& Technology, Gurdaspur, Punjab.

I had a experience of three and a half year of teaching. I had worked as a Asst. Proff. at G.N.D.U R.C. Gurdaspur for two years. Currently I am working as a Asst. Proff. at Sukhjindra College of Engg. \& Tech. Gurdaspur.

My paper IDCP: A new integrated fog removal algorithm using dark channel prior, Adaptive gamma correction \& Clahe has been accepted by the journal Asian Academic Research associates.

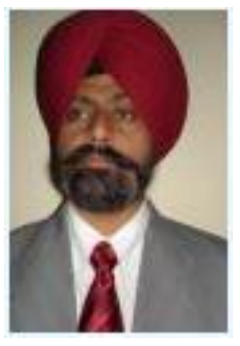

Gurpadam B. Singh is pursuing is Phd from Pec, Chandigarh. I am working as an Associate Professor at Beant College of Engineering \& Technology, Gurdaspur, Punjab.

Some of my publication like "Investigations of the phonemes of crow's using vector quantization" is published in GITAM Journal of Information communication Technology,

"Investigations of the phonemes in the calls of little owls using MFCC's". I had also delivered at delivered an Expert Lecture at ISTE approved Short Term Training Program "Digital Signal Processing: Concept and Applications" at Guru Nanak Dev Engineering College, Ludhiana on "Digital Filter Design" in Dec, 2006.

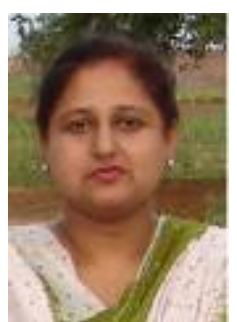

Mandeep C. Kaur was born on 3 april, 1972. I am working as a Asst. Proff. at Beant College of Engineering \& Technology, Gurdaspur, Punjab.

I had Attended a One week STTP from 16.2.2004 to 20.02.2004 on "Optical Fibre Technology and Applications" conducted by the Electronics and Communications Engineering Department at Beant College Of Engineering and Technology, Gurdaspur.( Approved by AICTE-ISTE). I had also attended One week STTP from 15-32004 to 19-3-2004 on "Modeling \& Simulation of Engineering and Environmental Systems" organized by the Department of applied Sciences, Humanities and Management at Beant College of Engineering and Technology, Gurdaspur. (Approved by AICTE-ISTE). 